\title{
Thermal Protection System and Trajectory Optimization for Orbital Plane Change Aeroassisted Maneuver
}

\author{
Antonio Mazzaracchio
}

\begin{abstract}
The aim of this paper was to identify, for a specific maneuver, the optimal combination between the trajectory and the associated heat shield configuration, namely the locations and thicknesses of the ablative and reusable zones, that maximize the allowable payload mass for a spacecraft. The analysis is conducted by considering the coupling between the trajectory's dynamics and the heat shield's thermal behavior while using a highly representative model of the heat shield. A global optimization procedure and original software were developed and implemented. The analyzed mission considers an aeroassisted transfer from two low Earth orbits with an assigned orbital plane change maneuver for a given delta wing vehicle equipped with a heat shield consisting of both ablative and reusable materials. The results indicate that the aeroassisted maneuver is more convenient than a "full propulsive" maneuver in the analyzed case, even considering the increased vehicle mass due to the presence of the heat shield.
\end{abstract}

KEYWORDS: Aeroassisted maneuver, Heat shield, Optimization, Orbital plane change, Thermal protection system.

\section{INTRODUCTION}

One of the most innovative concepts introduced in recent decades to meet the new, more stringent cost requirements of space missions is to make use of aeroassisted orbital maneuvers. These maneuvers can significantly reduce the propulsion requirements and travel time of a mission in favor of a higher available payload mass allocation.

Several studies have shown that using the atmosphere for assistance can benefit various classes of orbit (Walberg, 1985). Clearly, the spacecraft must be designed with an efficient aerodynamic configuration to properly utilize the atmosphere for the required global energy management.

Aeroassisted maneuvers are an extension of purely gravityassist maneuvers, but use a closer approach to a celestial body to encounter its atmosphere. Essentially, a gravity-assist maneuver depends on the size and mass of the planet and how closely it can be approached, whereas an aeroassisted maneuver depends also on the atmospheric properties and on the vehicle's aerodynamic characteristics.

A spacecraft, which necessarily encounters portions of atmospheric flight during a mission, must normally be equipped with a thermal protection system (TPS) to protect its structures, equipment, and payload from aerodynamic heating. The aerothermal conditions to which the vehicle is subjected are generally very severe and, in some cases, extreme.

The optimal design of a spacecraft and its trajectory is always a compromise between the conflicting interests of performance, safety and cost. Obviously, minimizing the TPS mass in compliance with the security constraints is a

1.Astronautical, Electrical and Energetic Engineering Department/Sapienza University of Rome - Rome - Italy

Author for correspondence: Antonio Mazzaracchio | Via Salaria 851 | 00138 Rome - Italy | E-mail: a_mazzaracchio@hotmail.com

Received: 06/12/12 | Accepted: 12/02/13 
primary requirement. In fact, any savings in the TPS mass can be translated into an increased payload mass. The problem becomes more structured when considering aeroassisted maneuvers, as it also takes into account the mass of propellant required during the various phases of the maneuver itself.

The search for increased benefits at the propulsive level from an aeroassisted maneuver implies greater use of the atmospheric phase of flight and a need for a more massive TPS. The question driving this paper is whether the increased TPS mass may cancel the propulsive benefits of the aeroassisted maneuver.

The TPS design is closely related to the trajectory that must be followed. It is evident that a change in the planned trajectory of the vehicle generates, from the perspective of global optimization, a variation to the TPS mass as a result of the modified aerothermal and environmental conditions.

The most critical parameters commonly considered in the design of a spacecraft's TPS are the "peak heat flux", the "peak dynamic pressure", and the "total heat load" (time integrated heat rate). The first two drive the choice of material for the TPS, and the last defines the thickness of the material. A fourth parameter (although not specific to the TPS) is the "peak deceleration", which is extremely important in the case of manned missions or payload limitations on the tolerable deceleration values.

During the phases of the mission in which they carry out their task of thermal protection, ablative TPS materials lose mass and thus engender a change in the shape of the vehicle. Similarly, changes that occur in the shape of the heat shield influence the vehicle's trajectory. Therefore, the dynamic and thermal problems are essentially coupled. However, they are generally treated separately because of the extremely high computational cost of solving the coupled problem. The usual procedure is, first, to optimize the trajectory and, then, to identify the configuration and the size of the heat shield that will ensure compliance with the imposed thermal constraints. More specifically, the optimized trajectory is first designed based on predefined heating limits. Then, a TPS able to withstand these thermal fluxes and thermal loads is designed. However, this decoupling technique generally leads to a nonoptimal solution. Additionally, simple behavioral models are commonly employed in the ablative analysis.

In this paper, the problem is instead solved by coupling the dynamic and thermal analyses. The detailed thermal analysis is performed at the stagnation point, and numerical and experimental approximations are used to calculate the heat entering the residual vehicle surface.

A new tool for trajectory and heat shield optimization is developed for the conceptual development of a spacecraft and its mission.

An aeroassisted maneuver executing a change of orbital plane between two circular orbits of the same radius is analyzed. The spacecraft is equipped with a heat shield consisting of ablative and reusable materials. The thermal models implemented are highly representative, and a genetic algorithm-based optimizer is used.

The remainder of this introduction provides a historical perspective on trajectory and TPS optimization methods. A review of the model and the governing equations of the problem is presented in next chapter. Afterwards, the optimization procedure is discussed along with the heat shield model adopted. A case study on a delta wing vehicle is presented, and the relevant results and analyses are discussed. The final chapter offers a summary, conclusions, and recommendations for future improvements.

A historical review of the methodologies used in the combined optimization of trajectories and TPSs is described by McGuire et al. (2004) and is briefly summarized hereinafter.

In 1974, Garcia and Fowler introduced a new parameter for optimizing the trajectory and TPS of the Space Shuttle. In place of the simple heat load, which is well known to be the major driver for the mass of the heat shield, they used an "objective function" expressed as an integrated function of the heating rate at the stagnation point and of the angle of attack, with limits on the surface temperature.

For the design of the HL-20 vehicle in 1993, Powell defined a guidance and control scheme to maintain a constant entering heat flux at the stagnation point by varying the bank angle but keeping the angle of attack constant. Powell also left the total heat load unconstrained. The trajectories obtained from this method were used by Wurster and Stone to calculate the relevant aerothermal database, which was in turn used as the main criterion in the selection of TPS materials.

In 1998, Hill et al. generated optimal trajectories for the X-33 using the temperatures of ten different zones of the vehicle as constraints. They employed an aerothermal database environment, created by Prabhu et al., using computational fluid dynamics (CFD) techniques. This comprehensive database was created by decoupling the thermal and dynamic problems regardless of the trajectory employed. 
In two independent works, also in 1998, both Windhorst et al. and Chou et al. generated optimal trajectories with the aim of minimizing the heat load at a specific point on the TPS surface.

Also in 1998, Allen et al. created an integrated multidisciplinary tool (consisting of aerothermodynamics, trajectory generation, and heat shield sizing modules) for the optimization of both trajectories and TPSs for planetary probes, in which the heat load was minimized, leaving the user to decide the TPS material distribution.

In 2001, Nishioka and Ogasawara, using a method similar to that introduced previously by Garcia and Fowler, derived an expression that depends on the geometry of the vehicle and minimizes the heat load in the reentry trajectories of the Space Shuttle.

Also in 2001, to generate optimal trajectories minimizing the mass of the TPS for a crew transfer vehicle (CTV), Saunders et al. adopted a methodology similar to that of Hill in 1998. They used several aerothermal performance constraint curves specific to each material. These curves correlated the altitude and speed of the vehicle so as not to exceed specific temperature limits, but left both the total heat load and the flight time unconstrained.

There is also a large body of literature that has addressed the problem from a multidisciplinary point of view that also involves the design of the vehicle. Examples of such approaches can be found in works by Menees (1983), Windhorst et al. (2004), and Joshua et al. (2008).

\section{MODEL AND GOVERNING EQUATIONS}

The aim of the present research, as already indicated, was to identify optimal trajectories, subject to heat flux constraints, as well as other possible limitations, while leaving the total heat load unconstrained. The objective is to minimize the sum of the masses of the TPS and the propellant necessary to accomplish the desired orbital maneuver. The vehicle has a given size, shape, and initial total mass, and it must carry out an assigned variation to the inclination of its orbital plane between two circular low Earth orbits (LEOs) of the same radius.

\section{AEROASSISTED ORBITAL PLANE CHANGE}

Figure 1 shows a classic scheme for an aeroassisted maneuver that changes the inclination of the orbital plane. The strategy is to use a combination of propulsive maneuvers in space and aerodynamic maneuvers in the atmosphere.

More precisely, one assumes that the propulsive phases of the maneuvers are all concentrated in three impulses, and, therefore, the part of the flight within the atmosphere takes place without the use of propulsion. It is also assumed that the change to the orbital inclination occurs entirely during the atmospheric portion of the flight.

The first propulsive impulse is a deorbit impulse, varying the speed by $\Delta V_{1}$ from the initial LEO of altitude $H_{A}$ to enter the atmosphere along an elliptic orbit segment. The second is a boost impulse upon exiting the atmosphere and is expressed by a speed variation of $\Delta V_{2}$ to achieve the final LEO by ascending once more along an elliptic orbit segment. The third and final impulse is a circularizing impulse, which varies the speed by $\Delta V_{3}$ to circularize the vehicle's path within the final LEO of altitude $H_{B}$, which is equal to $H_{A}$ by assumption.

It is useful to describe in more detail the various phases of the mission as follows. Initially, the vehicle is moving on a circular orbit of radius $R_{A}$ with a speed $V_{A}$, around the Earth,

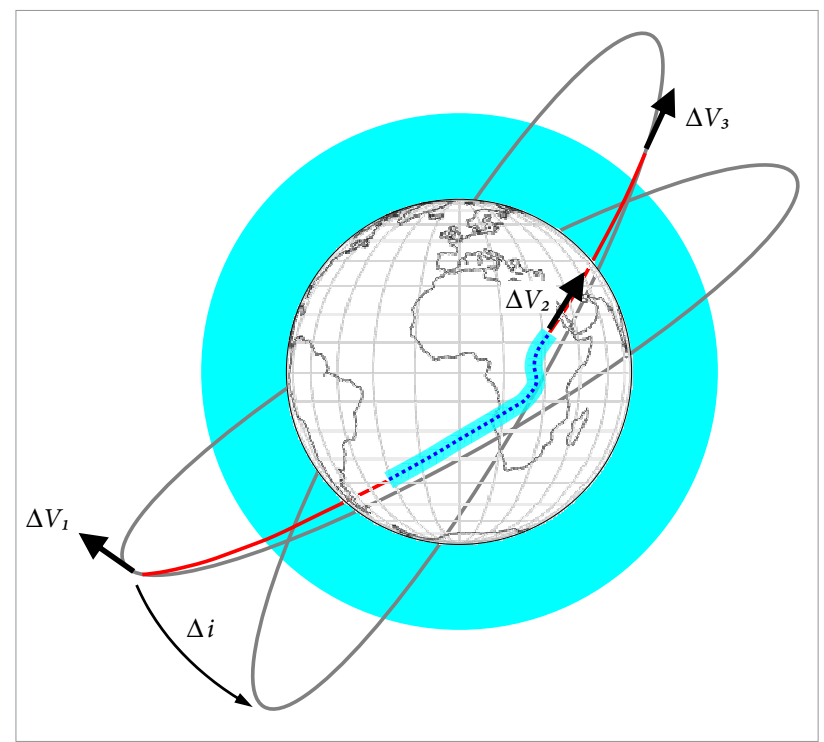

Figure 1. Schematic of aeroassisted orbital plane change maneuver. 
which has radius $R_{\oplus}$. The expression of the circular speed is the following:

$V_{A}=\sqrt{\mu / R_{A}}$

where:

$R_{A}=R_{\oplus}+H_{A}$

The deorbit is accomplished by applying the first impulse $\Delta V_{1}$ against the spacecraft's motion. This impulse places the vehicle along an elliptic orbit with the perigee inside the dense layers of the atmosphere. The atmospheric region below the altitude $H_{a t m}$, in which the aerodynamic effects are considered to be conventionally present, is denoted the "sensible atmosphere". One can determine the speed $V_{i}$ and the flight path angle $\gamma_{i}$ of the vehicle's trajectory at the atmospheric entry point $\left(H=H_{a t m}\right)$ as follows:

$V_{i}=\sqrt{2 \mu\left(\frac{1}{R_{a t m}}+\frac{\left(V_{A}-\Delta V_{1}\right)^{2}}{2}-\frac{1}{R_{A}}\right)}$

$\gamma_{i}=\cos ^{-1}\left[\frac{R_{A}}{R_{a t m} V_{i}}\left(V_{A}-\Delta V_{1}\right)\right)$

where:

$R_{a t m}=R_{\oplus}+H_{a t m}$

Obviously, it is necessary that the applied $\Delta V_{1}$ be greater than the minimum $\Delta V_{1, \min }$, for which there would be only a tangential trajectory to the edge of the sensible atmosphere. This $\Delta V_{1, \min }$ is given by the following expression:

$$
\Delta V_{i, \min }=\sqrt{\frac{\mu}{R_{A}}}-\sqrt{2 \mu \frac{\frac{1}{R_{a t m}}-\frac{1}{R_{A}}}{\left(\frac{R_{A}}{R_{a t m}}\right)^{2}-1}}
$$

During the atmospheric portion of flight, the vehicle performs the required change in orbital inclination, being optimally controlled by modulations of both the angle of attack $\alpha$ and the bank angle $\sigma$ while subject to the heating constraints. During this phase, the vehicle's speed decreases due to aerodynamic drag.
Because of the energy lost during the atmospheric crossing and the turn, a new impulse is necessary to achieve the final altitude. At the end of atmospheric flight, the vehicle is situated at an altitude $H_{\text {atm }}$ again, is driven at a speed $V_{u}$, and has a flight path angle equal to $\gamma_{u}$.

A second propulsion impulse (boost) is applied to enter an ascending elliptic orbit with the apogee equal to the radius of the final circular orbit (in this case, the same as the initial orbit). The required $\Delta V_{2}$, as a function of $V_{u}$ and $\gamma_{u}$, can be found from the following expression:

$\Delta V_{2}=\sqrt{2 \mu \frac{\frac{1}{R_{B}}-\frac{1}{R_{a t m}}}{1-\left(\frac{R_{B}}{R_{a t m}}\right)^{2} \cos ^{2} \gamma_{u}}}-V_{u}$

Once the final altitude is reached, the third impulse is applied to circularize the final orbit. The expression for the third $\Delta V$ is the following:

$\Delta V_{3}=\sqrt{\frac{\mu}{R_{B}}}-\sqrt{2 \mu \frac{\frac{1}{R_{B}}-\frac{1}{R_{a t m}}}{1-\left(\frac{R_{B}}{R_{a t m}}\right)^{2} \cos ^{2} \gamma_{u}}} \frac{R_{B}}{R_{a t m}} \cos \gamma_{u}$

\section{AERODYNAMIC HEATING}

The atmospheric flight reduces the vehicle's kinetic energy, which is mostly converted into the entering heat flux. This heat flux is evaluated at the stagnation point through correlations for both the convective and radiative components.

The Sutton-Graves correlation is used for the convective component of the heat flux:

$\dot{q}_{c o n}=C_{c}\left(\frac{\rho_{a t m}}{r_{n}}\right)^{1 / 2} V^{3}\left(1-\frac{h_{w}}{h_{0}}\right)$

and includes the "hot wall" correction factor $1-\left(h_{w} / h_{0}\right)$.

The radiative heat flux correlation is based on the TauberSutton relation:

$\dot{q}_{r a d}=C_{r} r_{n}^{a} \rho_{a t m}^{b} f(V)$ 
The value of the constant in Eq. 9 can be found in work by Havey (1982). The values of the constants in Eq. 10 and the tabulated function $f(V)$ are given by Tauber and Sutton (1991). The total heat flux at the stagnation point is then the sum of both the convective and radiative contributions:

$\dot{q}_{\text {tot }}=\dot{q}_{\text {con }}+\dot{q}_{\text {rad }}$

The heat flux is assumed to be independent of the angle of attack. Thus, the latter appears only in the guidance equations. This assumption is commonly acceptable for an engineering tool suitable for conceptual design phases (an example of this assumption can be found in a paper by Gogu et al., 2009).

\section{THERMAL MODEL}

The thermal analysis is performed with a onedimensional plane model (Fig. 2). The model for the ablative part of the heat shield and the relevant assumptions are thoroughly described in previous work by the author (Mazzaracchio and Marchetti, 2010). However, it is worth briefly recalling here the set of equations that form the basis of the model.

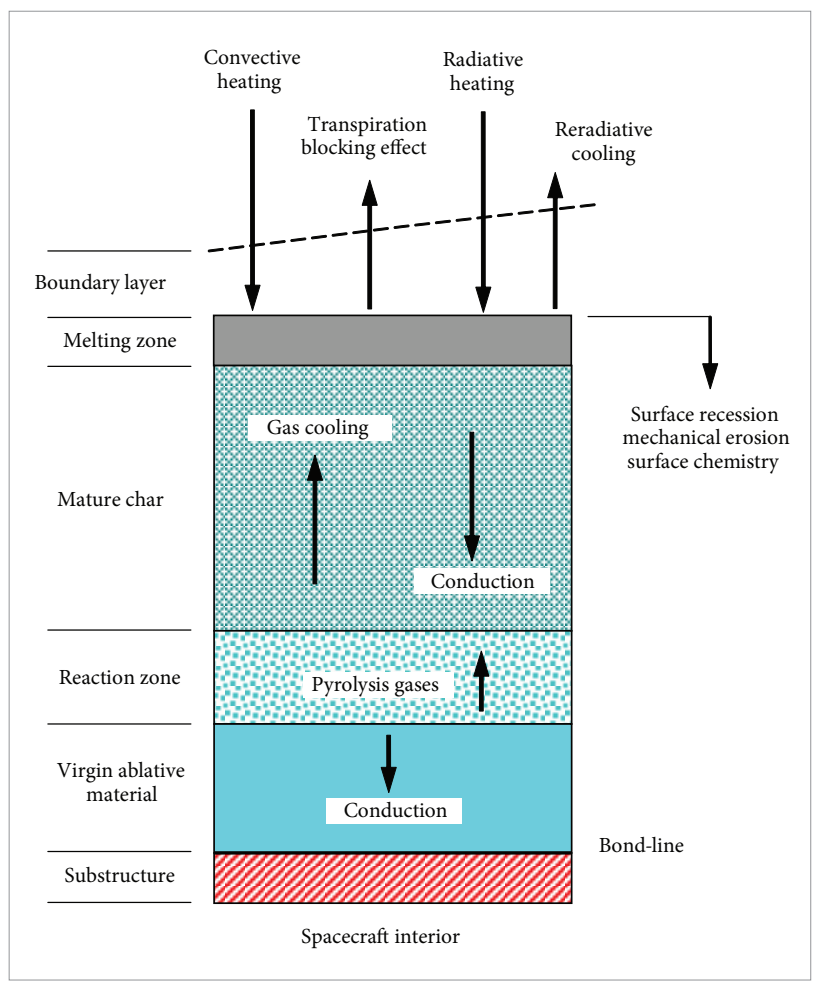

The internal energy balance is expressed as follows:

$$
\begin{array}{r}
\left.\rho c_{P} \frac{\partial T}{\partial t}\right|_{x}=\left.\frac{\partial}{\partial x}\left(k \frac{\partial T}{\partial x}-\dot{q}_{R}\right)\right|_{t}+\left.\left(h_{d}-\bar{h}\right) \frac{\partial \rho}{\partial t}\right|_{y}+ \\
\left.\dot{s}_{r} \rho c_{P} \frac{\partial T}{\partial x}\right|_{t}+\left.\dot{m}_{g} \frac{\partial h_{d}}{\partial x}\right|_{t}
\end{array}
$$

In Eq. 12 , the coordinate system $y$ is fixed, whereas the mobile coordinate system $x$ moves with the receding surface; initially, the two coincide. The terms in Eq. 12 each represent, in order: the rate of storage of thermal energy, the net rate of thermal energy transferred by conduction and internal radiation, the energy-consumption rate from pyrolysis, the convective energy rate due to coordinate system movement, and the convective rate from the pyrolysis gases. Because of the hypothesis of the internal opacity of the body, the $\dot{q}_{R}$ term is null.

For both virgin material and char, the local specific heat depends on temperature, whereas the local thermal conductivity depends on both temperature and pressure. The pyrolysis gas enthalpy $h_{d}$ also depends on temperature and pressure, whereas the quantity $\bar{h}$, which is the partial heat of charring, is defined as a function of both virgin and charred material properties as follows:

$$
\bar{h}=\frac{\rho_{v} h_{v}-\rho_{c} h_{c}}{\rho_{v}-\rho_{c}}
$$

The Arrhenius equation is used to represent the pyrolysis decomposition in a large-scale model:

$$
\frac{\partial \rho}{\partial t}=K_{c f}\left(\frac{\rho-\rho_{c}}{\rho_{v}-\rho_{c}}\right)^{n_{r}} \exp ^{-B_{T} / T}
$$

The internal decomposition transforms part of the solid ablative material into pyrolysis gases. Because of the assumptions of quasi-static one-dimensional flow and impermeability of the interface with the virgin material zone, the pyrolysis gases' mass flow is related to the decomposition by the following simple expression:

$$
\frac{\partial \dot{m}_{g}}{\partial y}=\frac{\partial \rho}{\partial t}
$$

Figure 2. Schematic of the ablation phenomena. 
The conditions at the external ablating surface are determined by the convective and radiative components of the aerodynamic heating and by the thermo-chemical interactions with the warm gases in the boundary layer. The energy balance at the surface is expressed in the following relation:

$\dot{Q}_{i n}=\dot{q}_{c, b l o w}+\dot{q}_{\text {rad }}+\dot{q}_{c o m b}-F \sigma_{S B} \varepsilon_{w}\left(T_{w}^{4}-T_{\infty}^{4}\right)$

The first term, $\dot{q}_{c, \text { blow }}$, comes from considering the reduction of the convective component by the injection of pyrolysis gases into the boundary layer. A second order approximation, a function of the mass flow of the outgoing gases known as "transpiration theory", is assumed to represent this "blocking effect".

Setting:

$A=\frac{h_{0}}{\dot{q}_{c, w}}\left(\alpha_{c} \dot{m}_{c}+\alpha_{g} \dot{m}_{g}\right)$

the final expression for the net convective heat flux is given by the following equations:

$$
\left\{\begin{array}{l}
A<2.25 \\
\dot{q}_{c, \text { blow }}=\left(1-0.724 A+0.13 A^{2}\right) \dot{q}_{c o n} \\
A \geq 2.25 \\
\dot{q}_{c, \text { blow }}=0.04 \dot{q}_{c o n}
\end{array}\right.
$$

The coefficients $\alpha_{c}$ and $\alpha_{g}$ are used to differentiate the molecular weight of the gases in the boundary layer from the molecular weight of the injected pyrolysis gases, respectively. The coefficient $\alpha_{c}$ also takes into account the fraction of char that is removed mechanically and not due to ablation.

In the presence of an oxidizing atmosphere, the heating due to combustion of the solid ablation products in the boundary layer must be considered, and complete combustion is assumed:

$\dot{q}_{c o m b}=\dot{m}_{c} \Delta h_{c o m b}$

Finally, the char recession rate $\dot{s}_{r}$ is obtained from experimental data as a function of either the surface temperature or the total entering heat flux.

As a consequence of char removal, the surface moves with respect to the fixed coordinate system. The distance between the initial position of the surface and the current position gives the thickness loss. This distance is given by:

$$
s_{r}=\int_{0}^{t} \dot{s}_{r} d t
$$

The model of the reusable portion of the heat shield is a classic model used for heat transfer without phase changes and surface recession, and was derived from the ablative model by eliminating all of the irrelevant phenomena due to the pyrolysis. Equations 12 and 16 become respectively:

$\left.\rho c_{P} \frac{\partial T}{\partial t}\right|_{x}=\left.\frac{\partial}{\partial x}\left(k \frac{\partial T}{\partial x}-\dot{q}_{R}\right)\right|_{t}$

$\dot{Q}_{i n}=\dot{q}_{c o n}+\dot{q}_{r a d}-F \sigma_{S B} \varepsilon_{w}\left(T_{w}^{4}-T_{\infty}^{4}\right)$

Obviously, the dependence from temperature and pressure of the thermal characteristics is preserved.

Equation 21 is also used for modeling the heat transfer in the substructure.

\section{ATMOSPHERIC FLIGHT}

The vehicle is considered to be moving subject to a Newtonian inverse square gravitational law, neglecting the Earth's rotation:

$g=\mu / R^{2}$

The three degree-of-freedom differential equations of motion in spherical coordinates are the following (Vinh et al., 1980):

$$
\begin{aligned}
& \frac{d R}{d t}=V \sin \gamma \\
& \frac{d \theta}{d t}=\frac{V \cos \gamma \cos \psi}{R \cos \varphi}
\end{aligned}
$$

$\frac{d \varphi}{d t}=\frac{V \cos \gamma \sin \psi}{R}$

$\frac{d V}{d t}=-\frac{D}{m}-g \sin \gamma$

$\frac{d \gamma}{d t}=\frac{1}{V}\left(\frac{L \cos \gamma}{m}-g \cos \gamma+\frac{V^{2}}{R} \cos \gamma\right)$ 
$\frac{d \psi}{d t}=\frac{1}{V}\left(\frac{L \sin \sigma}{m \cos \gamma}-\frac{V^{2}}{R} \cos \gamma \cos \psi \tan \phi\right)$

The aerodynamic lift and drag forces are given, respectively, by:

$L=m C_{L} \rho S V^{2} / 2$

$D=m C_{D} \rho S V^{2} / 2$

Assuming a parabolic drag polar, the drag coefficient of the vehicle can be written as:

$C_{D}=C_{D 0}+K_{D} C_{L}^{2}$

where the lift coefficient $C_{L}$ is a linear function of the angle of attack:

$C_{L}=C_{L, \alpha} \alpha$

and obeys the following limitation:

$0 \leq C_{L} \leq C_{L, \max }$

Concerning Eq. 33 and Eq. 34, it can be assumed that the vehicle always enters the atmosphere with the same attitude: zero incidence and a bank angle equal to $-180^{\circ}$ (upside down). The angle of attack cannot take negative values because the lift modulation is enacted through the bank angle. In fact, it should be noted that a negative $C_{L}$ value can result from either a negative pitch angle or from a vehicle flying upside down with a positive pitch angle. In this latter case, to get a downward force, one must fly upside down with a positive lift coefficient.

A non-negative flight path angle is required at the atmospheric exit to ensure the completion of the maneuver:

$\gamma_{u} \geq 0$

To evaluate the change in the orbital plane inclination, it is possible to use the following relationship between inclination, heading, and latitude:

$\cos i=\cos \varphi \cos \psi$
This expression can be evaluated using the values at the atmospheric exit, assuming an initial inclination of $0^{\circ}$, because, as suggested, the change of orbital plane is accomplished entirely during the atmospheric portion of the flight.

Finally, a fourth-order Runge-Kutta integration is employed in the trajectory computation.

\section{OPTIMIZATION}

Figure 3 shows a diagram of the procedure adopted here. The inputs to the method consist of the vehicle model and the requirements and constraints for the maneuver. The trajectory is integrated and the thermal analysis is performed as previously described.

The process for determining the minimum thickness of the TPS is iterative.

The optimization methodology used here is a genetic algorithm (GA) described hereafter.

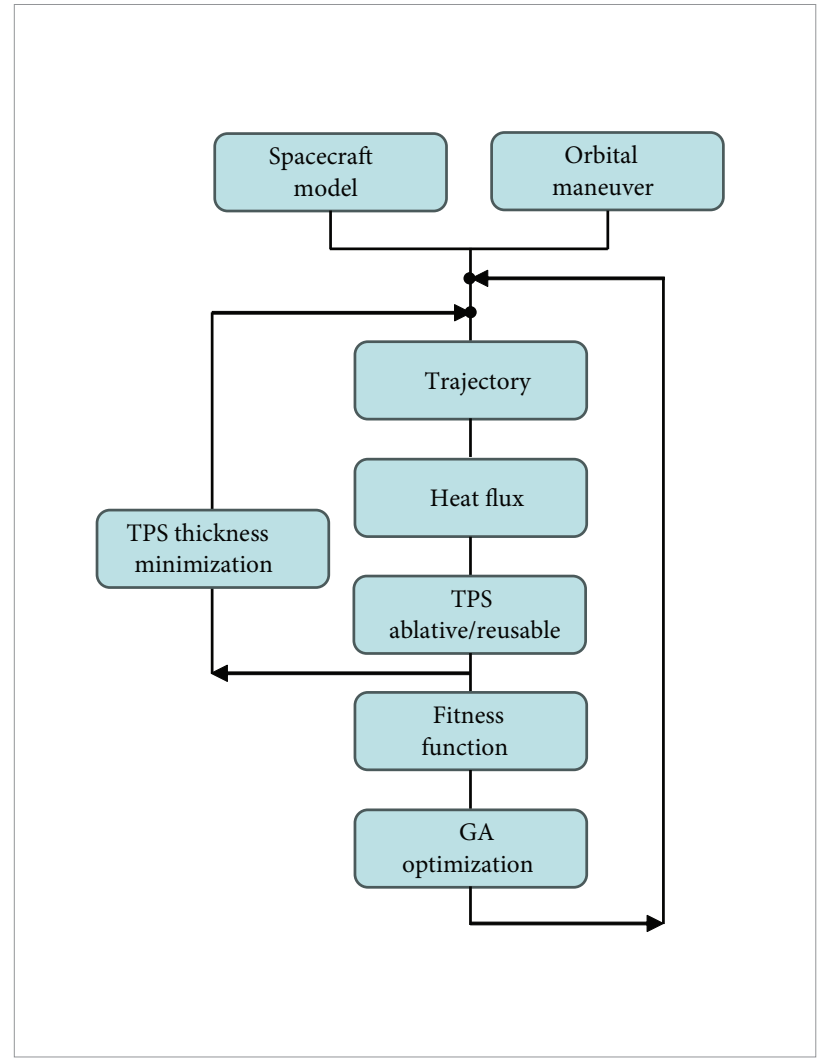

Figure 3. Diagram of optimization procedure. 


\section{GENETIC ALGORITHM}

Genetic algorithms, developed by Holland (1975), are a global search optimization method based on the fundamental principles of Darwinian evolution, such as natural selection and genetic mechanics.

Such a technique is - and has been - successfully used as a global optimization method for complex problems in many research fields and is also used as a suitable method for spacecraft trajectory optimization (Gath and Calise, 2001; Rajesh, 2002; Igarashi and Spencer, 2005). Other methods that are widely used in trajectory optimization problems because of their efficiency are the "gradient-based" optimizers.

The efficiency of these optimizers, however, is strongly influenced by the researcher's ability to provide good initial guesses and precise derivatives of the "fitness function". Without a good initial guess, the method may not converge or may produce a local minimum solution, which is often unacceptable. These fundamental aspects of the method involve a substantial degree of intervention by expert users.

Conversely, GA-based optimizers do not suffer from such limitations as they generate their own starting points. Thus, these optimizers are virtually insensitive to the initial guess. They are also intrinsically robust to non-linearities and discontinuities in the objective function. Their primary disadvantage is that GA-based optimizers require a large number of iterations and, subsequently, a longer computing time due to their generally slow convergence.

The following fact must be emphasized: a GA cannot find the optimal solution to a problem. Instead, it identifies the fittest individual in a population that has undergone a process of evolution for the improvement of the species. Evolution, even biological, does not aim to attain an optimum. The GA will use the population it has at its disposal to generate individuals that are above the average while also taking into account the constraints associated with the development. In other words, the GA does not perform a mathematical optimization in the strict sense of the term. Nevertheless, GAs are a highly robust and efficient optimization methodology.

The GA adopted here refers to a mixed one-point/twopoints crossover operator together with a reproduction plan that provides a full generational replacement with elitism (Charbonneau and Knap, 1995; Charbonneau, 2002a and 2002b).

The termination criterion corresponds to the maximum number of generations chosen and the main parameters adopted for the case studies presented here are summarized in Table 1.
The state variables chosen for the optimization procedure are: the time history of both the angle of attack and the bank angle, the $\Delta V_{1}$ used for deorbit, and, if required, the transit time in the atmosphere. In this study, the latter was left as a free parameter with an upper limit beyond which the mission is considered a failure. This assumption serves to exclude runs in which the vehicle flies by gliding up and down without enough energy to exit the atmosphere.

The fitness function is defined as the sum of an objective function (which must be maximized in the present case) plus the contributions of one "reward factor" for each constraint. Such a scheme allows easier handling of the constraints than the classic "penalty function" in a maximization problem such as this. In fact, the various reward factors are directly summable to the objective function.

The reward factor $R_{f, j}$ chosen here (Fig. 4), where ' $j$ ' indexes the various constraints, is a stepped pyramidal function (Yeniay, 2005). The function assumes a zero value outside of the range of definition (the base of the pyramid), which is defined by a semi-extension 'est-j' around the desired

Table 1. GA main parameters.

\begin{tabular}{|l|c|}
\hline Number of individuals in population & 100 \\
\hline Number of generations & 200 \\
\hline Number of genes & 5 \\
\hline Crossover probability & 0.85 \\
\hline Initial mutation rate & 0.005 \\
\hline Minimum mutation rate & 0.0005 \\
\hline Maximum mutation rate & 0.25 \\
\hline Relative fitness differential & 1.0 \\
\hline
\end{tabular}

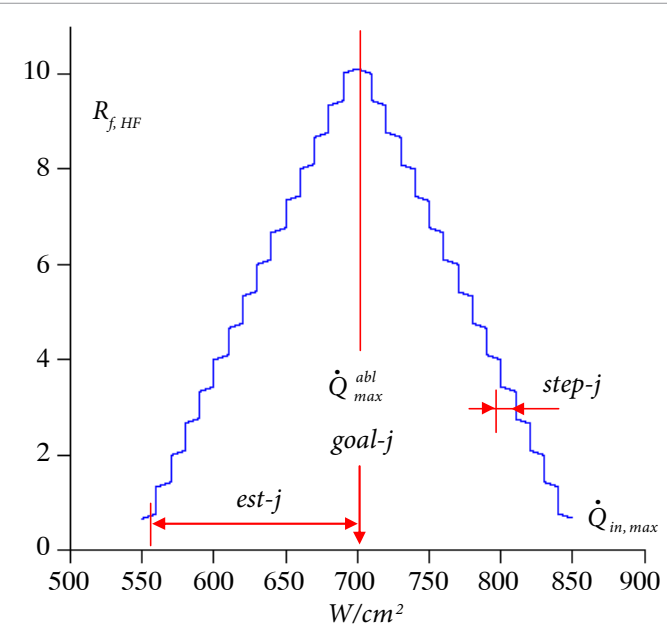

Figure 4. Example of the stepped pyramidal reward factor for the heat flux constraint. 
value of the constraint 'goal-j' ['goal-j' \pm 'est-j']. The range of definition is, in turn, divided into subintervals, each of which have an amplitude equal to 'step- $j$ ', and each characterized by a value of the "level of respect" (or, otherwise, the "level of violation") of the constraint that is proportional to the distance from ' $g o a l-j$ '.

This process allows the calculation of the first quantized contribution to the reward factor. A minor corrective addendum is then added within the same step. This addendum is a linear function of the distance from the boundary of the subinterval in question and generates the inclination of the step.

This structure of reward factors used in the genetic algorithm allows for a significant jump in value between one level and the next. It also allows the appropriate ordering of two points belonging to the same subinterval of merit. To choose the parameters defining the stepped pyramidal function, the value of the function at the end of a subinterval must not exceed the initial value of the next level. The additive contribution to the fitness function from the reward factors will grow as the variable values approach the values of the constraints, highlighting the individual in question within the entire population of the genetic algorithm. When all constraints are simultaneously satisfied, the value of the reward factor for this solution is enhanced.

\section{OBJECTIVE FUNCTION, CONSTRAINTS, AND FITNESS FUNCTION}

The total initial mass of the vehicle is the sum of the propellant mass, the TPS mass, and the structural and payload masses. The goal of the current optimization problem is to perform the orbital plane change while minimizing the sum of the mass of the propellant and the mass of the TPS needed. The objective function, which must be maximized, is then given by the final mass of the vehicle, that can be defined as the performance index of the problem.

Initially, the propellant consumption due to the deorbit impulse must be calculated. The vehicle's mass when it enters the atmosphere can be found by applying the Tsiolkovsky equation for the impulse:

$m_{v e, i}=m_{v e, i n i} e^{-\frac{\left|\Delta V_{1}\right|}{g_{0} I_{s p}}}$

This mass will be further reduced by $m_{T P S, ~ l o s}$ during the passage through the atmosphere because of TPS ablation.
The mass loss due to ablation comes from both surface recession, and the material density change due to pyrolysis. Thus, the mass of the vehicle at the atmosphere exit will be the following:

$m_{v e, u}=m_{v e, i}-m_{T P S, l o s}$

At this point, the boost and circularization impulses are applied in sequence, and the final vehicle mass is obtained by two successive applications of the Tsiolkovsky equation:

$m_{v e, f i n}=m_{v e, u} e^{-\frac{\left|\Delta V_{2}\right|+\left|\Delta V_{3}\right|}{g_{0} I_{s p}}}$

Compliance with the heat flux constraints and the required variation of the orbital inclination are ensured by the reward factors.

These reward factors are added to the objective function with appropriate multiplicative weights $w_{j}$, which are set to foster rapid convergence. The final expression of the fitness function $f f$ of the genetic algorithm is the following:

$f f=w_{m} \frac{m_{v e, f i n}}{m_{v e, i n i}}+w_{\Delta i} R_{f, \Delta i}+w_{H F} R_{f, H F}$

The convenience of the aeroassisted maneuver must be assessed with respect to the classic "all propulsive" maneuver in which the required inclination change is made by a single propulsive impulse outside of the atmosphere.

For circular orbits in which only the inclination is changed while all other orbital characteristics remain the same, the required $\Delta V$ is the following:

$\Delta V_{a p}=2 \sqrt{\frac{\mu}{R_{A}}} \sin \left(\frac{\Delta i}{2}\right)$

Consequently, the expression for the final mass of the vehicle is:

$m_{v e, \text { fin }, a p}=m_{v e, i n i} e^{-\frac{\left|\Delta V_{a p}\right|}{g_{0} I_{s p}}}$

The developed optimization method was validated by comparison with the trajectories presented by Gogu et al. (2009). 


\section{HEAT SHIELD MODEL}

The kinematic conditions of the mission and the properties of the atmosphere are such that the aerodynamic heating is significant. The use of a considerable heat shield is thus necessary, as is often highlighted.

Considering the spacecraft as a lifting vehicle, one assumes the presence of a protective coating on a portion of the outer surface of the vehicle. In particular, only the lower areas of the nose, body, and wings are covered by the TPS, corresponding to about $63 \%$ of the vehicle's total surface.

Due to the limited allowable maximal heat flux and surface temperature for the reusable materials, the severity of the thermal environment does not allow for the adoption of a fully reusable heat shield.

Conversely, the adoption of a fully ablative TPS may result in significant additional mass, as the density of most of these materials is considerably higher than the reusable ones. Moreover, due to the lower entering heat flux on some regions of the TPS, the adoption of ablative materials in these zones would be unnecessary. A hybrid TPS is thus adopted, in which the use of the reusable material is confined to the areas where the entering heat flux is lower than the specified operational limit $\dot{Q}_{\max }^{\text {reu }}$.

\section{MAPPING AND DIMENSIONAL EVALUATION OF TPS}

The mapping and sizing of the TPS are carried out through the procedure detailed as follows (Fig. 5(a) and Fig. 5(b)).

- The total area of the vehicle is calculated as the sum of the contributions of the nose cone, the body and the two wings.

- The total area of the heat shield is reduced by a certain percentage from the total area of the vehicle to reflect the actual coverage of the TPS (Gogu et al., 2009).

- A thermal safety factor (TSF) is multiplied to the calculated thickness of the TPS materials.

- The nose cone is divided into spherical segment panels, the body into cylindrical panels and the wings into rectangular panels.

- For each panel, a reduction factor for the entering heat flux is calculated with respect to the stagnation point flux $\dot{Q}_{i n}$. For the nose cone and the body, this reduction is only considered longitudinally. For the wings, this reduction factor is expressed as a product of a transverse factor (on the leading edge) and a longitudinal factor. Figures 6(a) and 6(b) show the longitudinal and transverse normalized reduction factors taken from a work by Reuther et al. (2004) and slightly modified here.

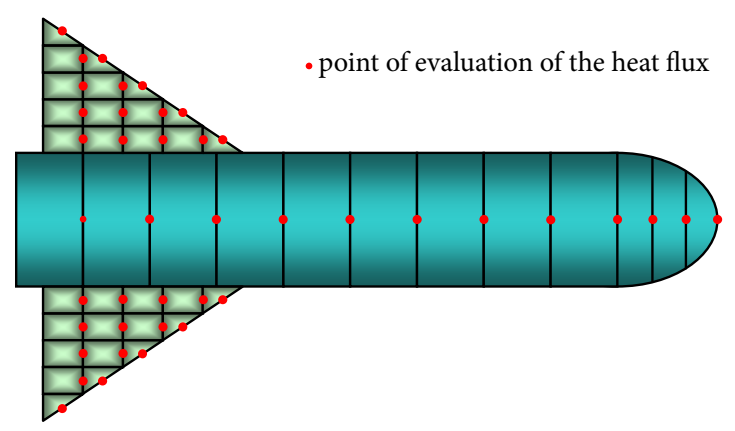

(a). TPS panels schematic.

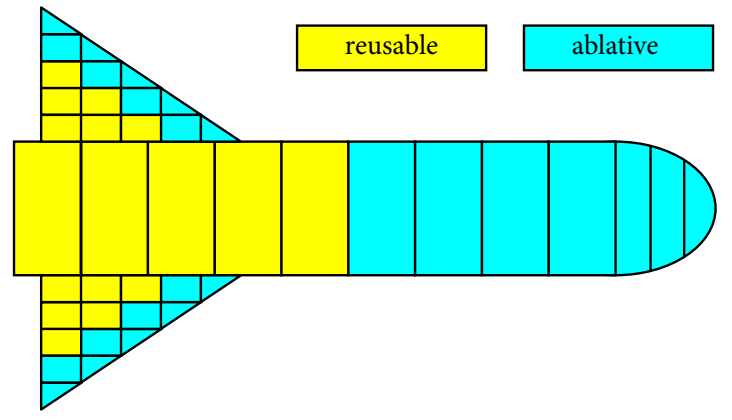

(b). Ablative versus reusable TPS areas.

Figure 5. Schematic of TPS model.

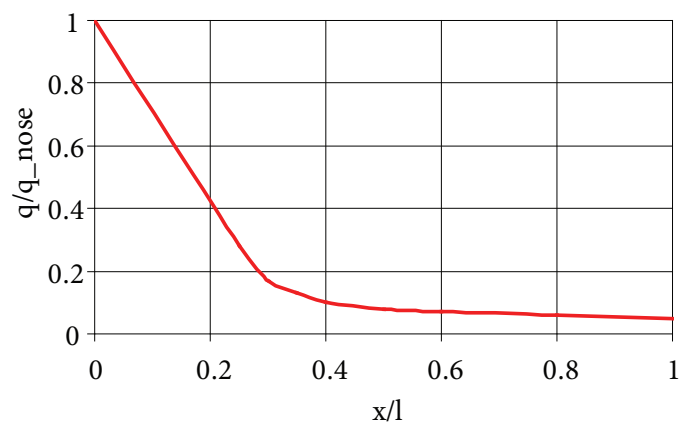

(a). Normalized heat reduction factor: longitudinal [nose and body].

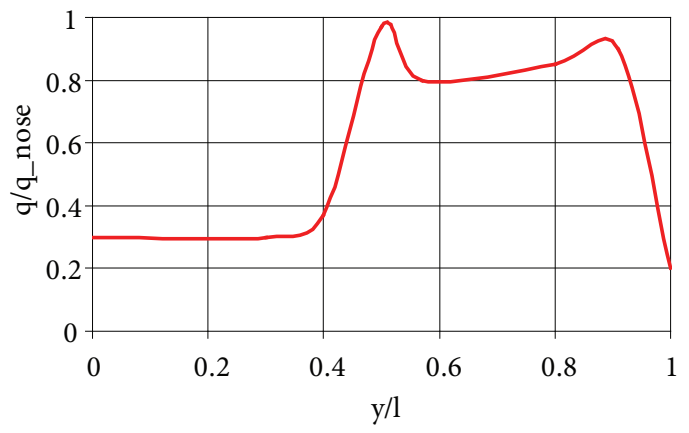

(b). Normalized heat reduction factor: transverse [wings].

Figure 6. Normalized heat reduction factor. 
- To be conservative, the reduction factor is calculated in the middle of the upper edge of each panel (point of maximal entering heat flux).

- The type of material used for each panel, reusable or ablative, is chosen depending on the level of the entering heat flux compared to the value of $\dot{Q}_{\text {max }}^{\text {reu }}$.

- Each panel contributes to the mass per unit area of the TPS in proportion to its thickness and type of material.

- The required thickness of each panel is considered to be directly proportional to the entering heat flux. This assumption allows a "reduced virtual equivalent surface" to be obtained as the sum over panels of the product of each panel surface with its respective reduction factor.

- For the reusable area, this reduction factor is again divided by the ratio between $\dot{Q}_{\max }^{r e u}$ and $\dot{Q}_{\max }^{a b l}$. In fact, in this instance, the initial thickness is equal to the maximum thickness of the reusable material itself.

Finally, in evaluating the mass, the adopted scheme is equivalent to a TPS with a uniform thickness equal to the maximum, including the TSF, but distributed on a "reduced virtual equivalent surface".

At this point, a methodological clarification is needed. For maximal savings in the TPS mass, as already indicated, the reusable material is assumed to cover all of the areas where the values of heat flux permit. In all other zones, the ablative material will be employed. Nevertheless, the zones where the reusable material can be used are still not defined.

The entering heat flux at the stagnation point, calculated from Eq. 11, is a function of the trajectory. For this reason, and because of the correlation with the heat flux entering the other areas of the vehicle, a mapping of the TPS in terms of reusable vs. ablative material is not definable at the beginning. However, at the end of the genetic evolutionary process, an optimal trajectory will be found that obeys the constraints imposed on the heat flux, among others. Thus, a TPS mapping consistent with this final situation can be adopted a priori. Then, as a result of these assumptions, the distribution of the entering heat flux is fixed in advance, making it possible to define the boundaries of the reusable material.

Obviously, during the search for the optimal trajectory, in all of the runs in which the heat flux constraints are not respected, an inappropriate TPS configuration will be achieved. This is especially true for runs in which the heat flux value is greater than the constraint. In these cases, a part of the reusable heat shield will be overexposed to entering heat flux, implying partial incompliance with the constraint value $\dot{Q}_{\max }^{\text {reu }}$. Conversely, if the vehicle will be exposed to a smaller heat flux than the constraint, the ablative material will be used more than necessary, covering areas that could be covered with the reusable material.

Note that in the unconstrained case, and precisely because of the absence of any indication concerning the entering heat flux, one assumes that the entire shield is ablative.

Returning again to the operational details, the design parameter chosen for sizing the TPS is the temperature of the bond-line $\left(T_{B L}\right)$, i.e., the temperature of the adhesive junction layer between the heat shield and the substructure. It is required that $T_{B L}$ be within the specified design temperature. The required minimum thicknesses are determined by independently iterating on both types of material. The minimum thickness of the ablative material is determined at the stagnation point, whereas the minimum thickness of the reusable material is calculated at the points with an entering heat flux equal to its operational limit.

The actual thicknesses of all other points of the heat shield are then considered to be linear with the local heat load. Therefore, because the exposure time is the same for all points, the required thicknesses are ultimately assumed to be linear with the maximum heat flux entering the point.

Note that during the iterative process to determine the minimum thickness within a single run, the trajectories do not vary noticeably from one iteration to another because the initial mass is constant by definition. In effect, the savings in the TPS mass due to the decreased thickness of the heat shield are offset by the mass of the structures and the payload so as to leave the initial total mass of the vehicle unchanged.

The developed thermal model and sizing tool were validated by the author (Mazzaracchio and Marchetti, 2010) by comparison with an industry standard high-fidelity ablation and thermal response program, namely the "Fully Implicit Ablation and Thermal" (FIAT) software of NASA Ames Research Center.

\section{CASE STUDY}

\section{SPACECRAFT}

The analyzed spacecraft was characterized by a delta-wing configuration with a high $L / D$, roughly similar to the Boeing X-37 vehicle. The dimensions and aerodynamic characteristics used were taken in part from Gogu et al. (2009) and NASA 
Facts (2003). Other data were reasonable assumptions made by the author. The primary dimensions and characteristics of the vehicle are listed in Table 2, and the principal aerodynamic and propulsive parameters are listed in Table 3.

\section{ATMOSPHERIC FLIGHT}

Table 4 presents the orbit altitudes of the required maneuver and the assumed conventional limit of the Earth's sensible atmosphere.

The atmospheric properties were derived from the 1976 US Standard Atmosphere model.

\section{TPS MATERIALS}

The spacecraft had a supposed heat shield consisting of a material called phenolic impregnated carbon ablator, known as PICA-15, for the ablative zones and LI-900 for the reusable zones.

PICA-15 was used in the Stardust probe, whose reentry capsule returned to Earth in 2006 with samples of cosmic and cometary dust collected during its mission. PICA is a relatively recent material that is characterized by a low density - well below that of the classic carbon phenolic - while at the same time having a high ablative capability for elevated heat fluxes. PICA also features a thermal conductivity lower than that of other ablative materials for equal entering heat fluxes. PICA consists of chopped carbon fibers as insulation with a phenolformaldehyde resin as an infiltrant. The high porosity of the composite is the reason for its low density and thermal conductivity (Tran et al., 1997).

LI-900 is a typical reusable material designed for use on the Space Shuttle TPS. LI-900 comes in the form of a surface insulating tile and is used to minimize thermal conductivity while ensuring maximum resistance to thermal shock. It is made from $99.9 \%$ pure silica glass fibers, which occupy only $6 \%$ of the total volume (Williams and Curry, 1992).

Both the ablative and reusable layers are bonded onto the substructure, which consists of a $12.7 \mathrm{~mm}$ thick carbon-carbon/aluminium honeycomb sandwich. The substructure is considered an integral part of the TPS.

\section{RESULTS AND ANALYSIS}

The study was conducted by analyzing three different cases corresponding to three different values for the entering heat
Table 2. Vehicle's dimensions and characteristics.

\begin{tabular}{|l|c|c|}
\hline Gross vehicle mass & $m_{v e, \text { ini }}$ & $4898.7 \mathrm{~kg}$ \\
\hline Vehicle length & $l_{v e}$ & $9.38 \mathrm{~m}$ \\
\hline Vehicle body radius & $r_{b}$ & $1.00 \mathrm{~m}$ \\
\hline Vehicle wing span & $w s_{v e}$ & $4.50 \mathrm{~m}$ \\
\hline Vehicle wing cord & $w c_{v e}$ & $3.50 \mathrm{~m}$ \\
\hline Vehicle reference surface & $S$ & $11.69 \mathrm{~m}^{2}$ \\
\hline Vehicle TPS total surface & $S_{T P S, v e}$ & $42.65 \mathrm{~m}^{2}$ \\
\hline Bond-line limit temperature & $T_{B L, l i m}$ & $450 \mathrm{~K}$ \\
\hline Thermal safety factor & $T S F$ & 1 \\
\hline
\end{tabular}

Table 3. Vehicle's aerodynamic and propulsive characteristics.

\begin{tabular}{|l|c|c|}
\hline Zero-lift drag coefficient & $C_{D 0}$ & 0.032 \\
\hline Induced drag factor & $K_{D}$ & 1.4 \\
\hline Lift coefficient derivative & $C_{L, \alpha}$ & 0.5699 \\
\hline Maximum lift coefficient & $C_{L, \max }$ & 0.4 \\
\hline Propellant specific impulse & $I_{s p}$ & $310 \mathrm{~s}$ \\
\hline
\end{tabular}

Table 4. Maneuver's characteristics.

\begin{tabular}{|l|c|c|}
\hline Initial LEO altitude & $H_{A}$ & $185.2 \mathrm{~km}$ \\
\hline Final LEO altitude & $H_{B}$ & $185.2 \mathrm{~km}$ \\
\hline Required inclination change & $\Delta i$ & $18^{\circ}$ \\
\hline Atmosphere's upper limit & $H_{a t m}$ & $129.6 \mathrm{~km}$ \\
\hline
\end{tabular}

flux constraint. The first two cases corresponded to $\dot{Q}_{\max }^{a b l}$ equal to 397 and $568 \mathrm{~W} / \mathrm{cm}^{2}$, which are equivalent, respectively, to 350 and $500 \mathrm{Btu} /\left(\mathrm{ft}^{2} \cdot \mathrm{s}\right)$. The third case was unconstrained.

The study required a running time of approximately 160 days on a I7 Intel ${ }^{\circledR}$ processor with 8 threads and a clock speed of $3.2 \mathrm{GHz}$. The fittest case was identified from a population of about 3.5 million individuals.

The "all propulsive" case for this mission provided, by mean of Eqs. 41 and 42 , a value for $\Delta V_{a p}$ equal to $2438.20 \mathrm{~m} / \mathrm{s}$, which implies a consumption of $2703.51 \mathrm{~kg}$ of propellant and a net vehicle mass equal to $2195.19 \mathrm{~kg}$.

Table 5 shows a comparison of the main characteristics of the obtained optimal trajectories and presents the maximum values of the dynamic pressure and the load factor. These latter are presented only for verification purposes, as they are not subject to constraints; in any case, their observed values are compatible with the feasibility of the mission.

All cases were characterized by similar values of $\Delta V_{1}$ and $\gamma_{i}$, whereas $\Delta V_{2}$ and $H_{\min }$ decreased with increasing $\dot{Q}_{\max }^{a b l}$. Concurrently, the flight time $t_{f}$ and the total heat load $H L$ increased with increasing $\dot{Q}_{\max }^{a b l}$. 
Table 5. Trajectory comparison.

\begin{tabular}{|l|c|c|c|}
\hline $\begin{array}{l}\text { Heating constraints } \\
\dot{Q}_{\max }^{\text {abl }}, \mathrm{W} / \mathrm{cm}^{2}\end{array}$ & $\mathbf{3 9 7}$ & $\mathbf{5 6 8}$ & No Limit \\
\hline$\dot{Q}_{i n, \max }, \mathrm{W} / \mathrm{cm}^{2}$ & 397.00 & 568.00 & 668.66 \\
\hline$\Delta V_{1}, \mathrm{~m} / \mathrm{s}$ & 31.74 & 30.24 & 31.73 \\
\hline$\Delta V_{2^{\prime}}, \mathrm{m} / \mathrm{s}$ & 1349.22 & 1278.05 & 1227.93 \\
\hline$\Delta V_{3^{\prime}}, \mathrm{m} / \mathrm{s}$ & 65.57 & 58.01 & 71.76 \\
\hline$t_{f l^{\prime}} \mathrm{s}$ & 1579.17 & 1616.80 & 1642.28 \\
\hline$H_{\min }, \mathrm{km}$ & 44.07 & 40.03 & 37.73 \\
\hline$\gamma_{i}{ }^{\circ}$ & -0.46 & -0.44 & -0.46 \\
\hline$\gamma_{u^{\prime}}{ }^{\circ}$ & 0.84 & 0.77 & 0.89 \\
\hline$H L, \mathrm{~J} / \mathrm{cm}^{2}$ & 72471 & 88448 & 88620 \\
\hline$q, \mathrm{kN} / \mathrm{m}^{2}$ & 57.75 & 104.81 & 143.77 \\
\hline$n, \mathrm{~g}$ & 4.33 & 5.46 & 5.05 \\
\hline
\end{tabular}

Table 6 presents the main differences among the three optimal TPS scenarios. As previously stated, a fully ablative heat shield is used in the unconstrained case. In this "not limited" case, the maximum observed heat flux is equal to $668.66 \mathrm{~W} / \mathrm{cm}^{2}$, which is much lower than the operating limit of the employed material (PICA-15). As expected, the thickness of the ablative part, and consequently its mass, grow as the heat load increases; in contrast, the overall mass of the TPS decreases considerably. This apparent contradiction lies in the type of materials used. Obviously, as the heating constraint takes higher values, the ablative portion of the heat shield is extended.

However, in the present work, which uses a low density ablative material, it is disadvantageous to use the reusable materials. In fact, even though they have a lower density, they require greater thicknesses. These factors combine to produce a lighter TPS when a fully ablative configuration is employed. Clearly, this behavior is not general, and different results are possible when denser ablative materials are used.

The mass lost during the crossing of the atmosphere due to the pyrolysis phenomena should be noted. Its value in the unconstrained case is $48 \%$ of the entire ablative part, $36 \%$ of the entire TPS mass, and $2 \%$ of the gross vehicle mass. The surface recession is about $7 \mathrm{~mm}$. Finally, in this unconstrained case, the mass fraction of the entire TPS is approximately $5.5 \%$ of the gross vehicle mass. Additionally, at the propulsive level, the total consumption of propellant also diminishes with the increasing value of the heating constraint.

The unconstrained case is therefore the most beneficial case in terms of savings in both the propellant mass and TPS mass, when using these particular TPS materials. To obtain the maximum benefit, in principle, one must adopt the highest
Table 6. Heat shield comparison.

\begin{tabular}{|c|c|c|c|}
\hline $\begin{array}{l}\text { Heating constraints } \\
\dot{Q}_{\max }^{a b l}, \mathbf{W} / \mathrm{cm}^{2}\end{array}$ & 397 & 568 & No Limit \\
\hline$m_{T P S}, \mathrm{~kg}$ & 308.66 & 284.81 & 267.37 \\
\hline$m_{T P S}^{a b l}, \mathrm{~kg}$ & 164.76 & 190.20 & 198.19 \\
\hline$m_{T P S}^{r e u}, \mathrm{~kg}$ & 74.72 & 25.43 & - \\
\hline$m_{T P S}^{s s}, \mathrm{~kg}$ & 69.18 & 69.18 & 69.18 \\
\hline$s_{r}, \mathrm{~mm}$ & 3.69 & 6.70 & 7.07 \\
\hline$m_{T P S, ~ l o s}, \mathrm{~kg}$ & 76.86 & 91.83 & 95.34 \\
\hline$m_{T P S, \text { prop }}, \mathrm{kg}$ & 1827.37 & 1741.88 & 1705.30 \\
\hline$\delta_{T P S}^{a b l}, \mathrm{~mm}$ & 87.50 & 89.79 & 90.34 \\
\hline$\delta_{T P S}^{r e u}, \mathrm{~mm}$ & 32.15 & 31.49 & - \\
\hline $\begin{array}{l}\text { Mass gain wrt "all } \\
\text { propulsive" case, } \mathrm{kg}\end{array}$ & 567.48 & 676.82 & 730.84 \\
\hline Gain wrt $m_{v e, \text { ini }}, \%$ & 11.58 & 13.82 & 14.91 \\
\hline
\end{tabular}

allowable heating constraint. However, in this case, higher load factors and dynamic pressures are to be expected and may restrict the maximum allowable heat flux. In Fig. 7, a comparison of all of the masses involved highlights the benefit of this maneuver in comparison with the "all propulsive" case. The unconstrained case allows a considerable payload mass gain of $730.84 \mathrm{~kg}$, which corresponds to about $15 \%$ of the gross vehicle mass.

Figures $8,9,10$, and 11 show the altitude, the velocity, the total entering heat flux, and the vehicle's mass, respectively, as functions of the flight time during the atmospheric pass.

When the lowest thermal constraint is imposed, the vehicle must dive into the atmosphere earlier to perform the assigned maneuver, even if it will descend less than in the other cases (Fig. 8). More precisely, to achieve the desired change in the orbital plane, it will fly for a longer time in less dense layers of the atmosphere than in the cases with a higher flux limit. This is necessary to prevent violation of the heat constraint and because of the lower

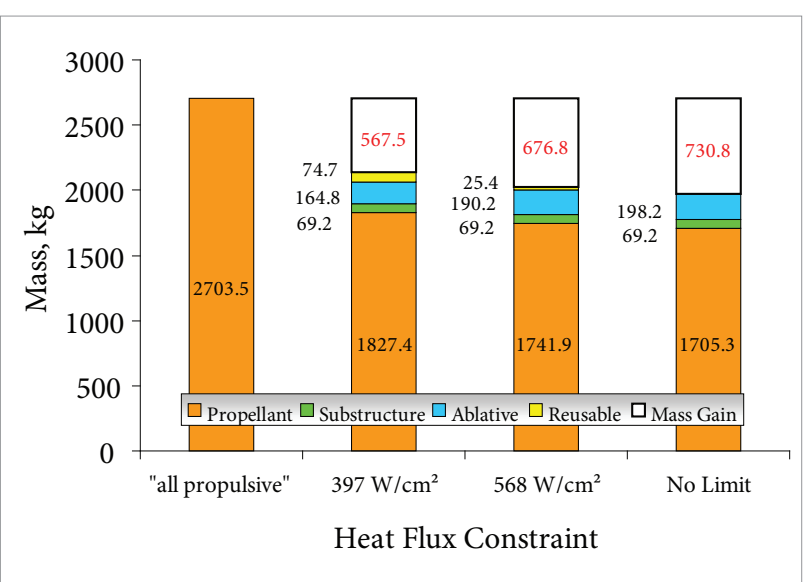

Figure 7. Mass comparison. 
available maneuverability in this region. However, the total flight time is ultimately greater for the unconstrained case. This fact and the lower minimum altitude reached, makes the total heat load for this trajectory higher. Figure 9 shows that the reduction in the velocity is less important in the unconstrained case, justifying the requirement of a smaller $\Delta V_{2}$ at the atmosphere exit.

Figures 12, 13, and 14 represent the coverage areas, the type of material and the thicknesses of the heat shield, respectively, for all three cases on a half-vehicle scheme. It should be noted that the assumptions have led to a theoretical thickness step on the border between the reusable and ablative parts.

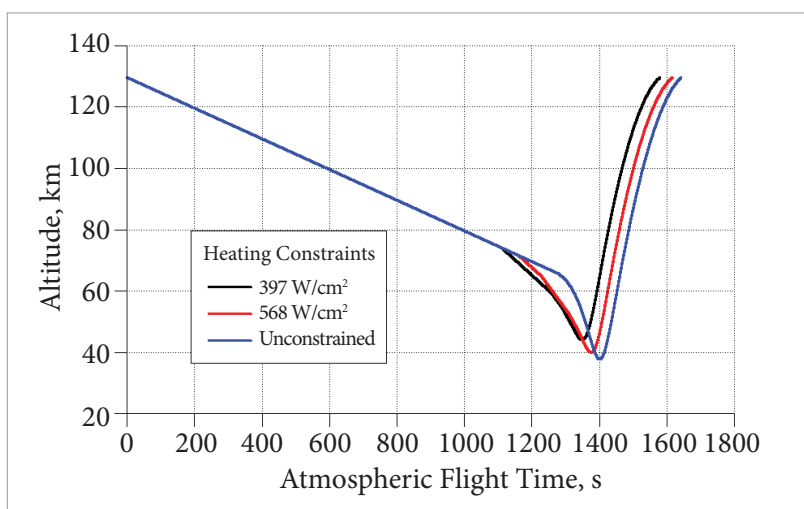

Figure 8. Altitude versus time.

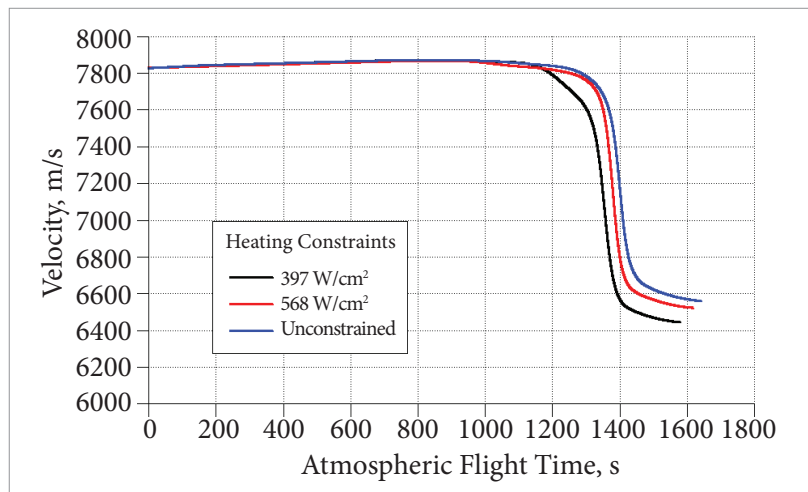

Figure 9. Velocity versus time.

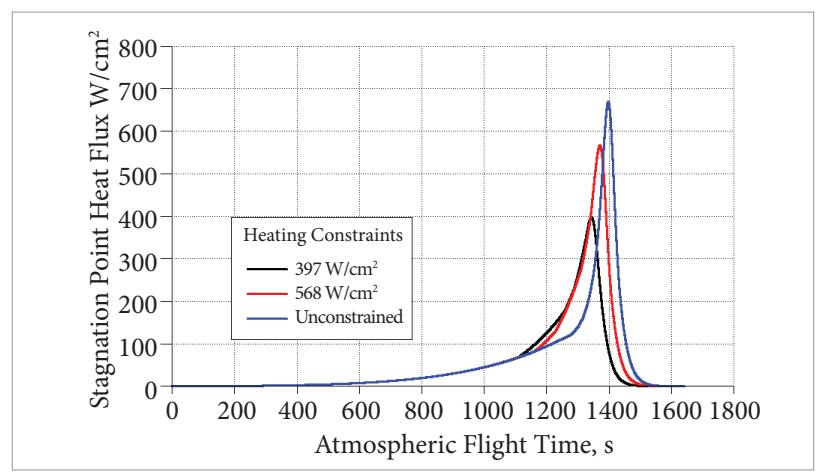

Figure 10. Total entering heat flux versus time.

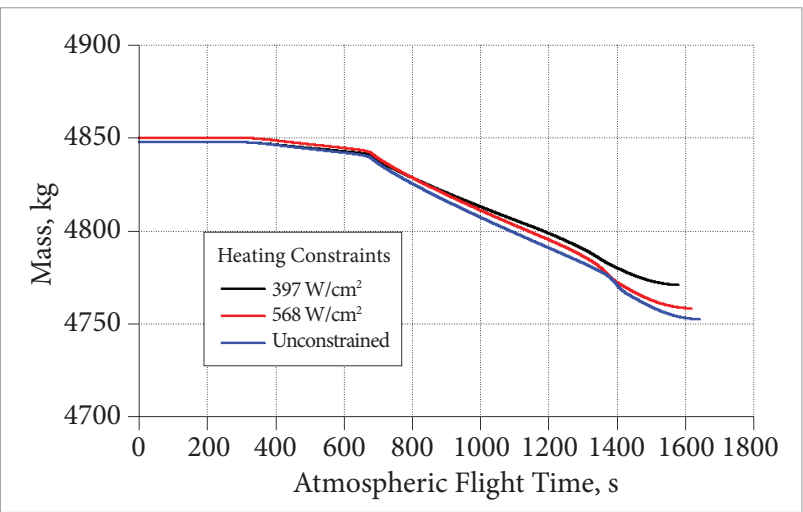

Figure 11. Mass versus time.

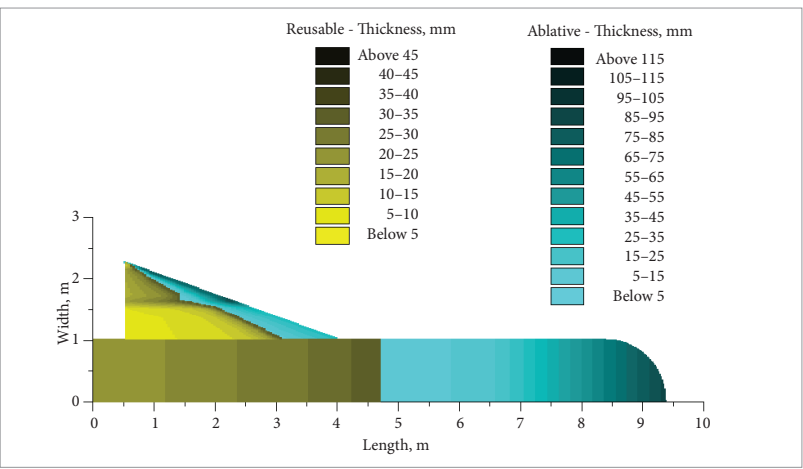

Figure 12. TPS configuration for the case with $\dot{Q}_{\max }^{a b l}=397 \mathrm{~W} / \mathrm{cm}^{2}$.

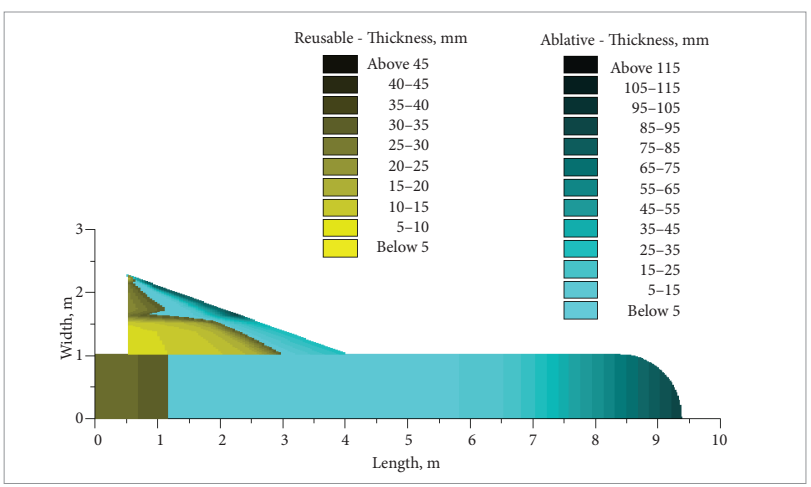

Figure 13. TPS configuration for the case with $\dot{Q}_{\max }^{a b l}=568 \mathrm{~W} / \mathrm{cm}^{2}$.

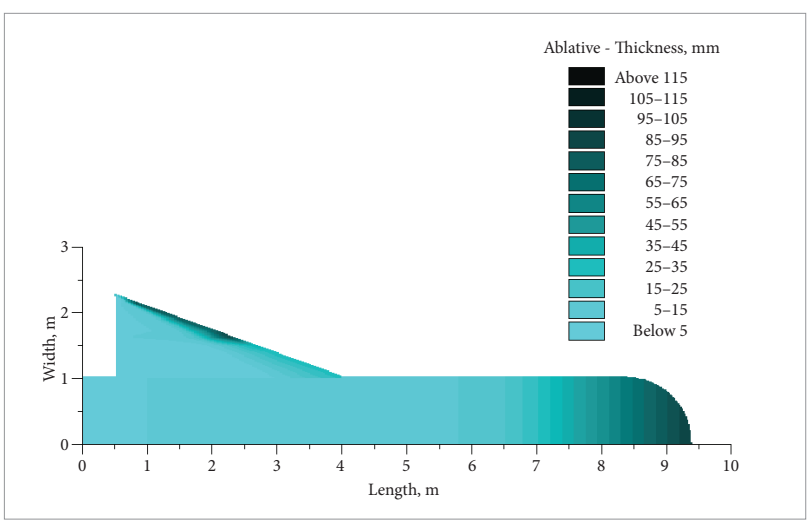

Figure 14. TPS configuration for the unconstrained case. 


\section{SUMMARY AND CONCLUSION}

A new tool was developed for the optimization of aeroassisted maneuvers by coupling the dynamic and thermal problems, and taking into account the mass of the vehicle's TPS. This tool, which is suitable for conceptual design activities, refers to highly representative thermal models for reusable and ablative materials, and is based on a genetic algorithm optimizer.

A maneuver to change the orbital plane between two LEOs with the same radius was analyzed, taking the maximum allowable entering heat flux at the vehicle's stagnation point as a parameter.

The optimal trajectories for completing the specified mission were found along with the respective optimal configurations of the TPS.
The results of this case study indicate that the aeroassisted technique is much more convenient than "all propulsive" maneuvers, even when considering the increased mass due to the need to give the vehicle a heat shield. In fact, the total budget between the savings in the propellant mass and the increased TPS mass was positive and allows for a substantial increase in the payload mass of the vehicle. The most favorable case is the one with no limit on the entering heat flux and a full ablative heat shield.

The most important future developments and improvements are the following:

- Extension of the analysis to other types of missions.

- Extension of the analysis to other types of materials.

- Improvement of the genetic optimizer by coupling it with other optimization methods for faster convergence.

\section{REFERENCES}

Charbonneau, P., 2002a, "An introduction to genetic algorithms for numerical optimization", Boulder, Colorado.

Charbonneau, P., 2002b, "Release notes for Pikaia 1.2”, Boulder, Colorado.

Charbonneau, P. and Knap, B., 1995, "A user's guide to Pikaia 1.O", Boulder, Colorado.

Gath, P.F. and Calise, A.J., 2001, "Optimization of launch vehicle ascent trajectories with path constraints and coast arcs", Journal of Guidance, Control and Dynamics, Vol. 24, No. 2, pp. 296-304. doi: 10.2514/2.4712.

Gogu, C., Matsumura, T., Haftka, R.T. and Rao, A.V., 2009, “Aeroassisted orbital transfer trajectory optimization considering thermal protection system mass", Journal of Guidance, Control and Dynamics, Vol. 32, No. 3, pp. 927-938. doi: 10.2514/1.37684.

Havey, K.A. Jr., 1982, "Entry vehicle performance in low-heat-load trajectories", Journal of Spacecraft and Rockets, Vol. 19, No. 6, pp. 506512. doi: 10.2514/3.62293.

Holland, J.H., 1975, "Adaptation in natural and artificial systems", University of Michigan Press, Ann Arbor, Ml.

Igarashi, J. and Spencer, D.B., 2005, "Optimal continuous thrust orbit transfer using evolutionary algorithms", Journal of Guidance, Control and Dynamics, Vol. 28, No. 3, pp. 547-549. doi: 10.2514/1.11135.

Joshua, E.J., Lewis, M.J. and Starkey, R.P., 2008, "Analysis of optimal Earth entry heat shield/trajectory configurations", 15 Space Planes and Hypersonics Systems and Technologies Conference, Dayton, Ohio, paper no. AIAA-2008-2594

Mazzaracchio, A. and Marchetti, M., 2010, "A probabilistic sizing tool and Monte Carlo analysis for entry vehicle ablative thermal protection systems", Acta Astronautica, Vol. 66, issues 5-6, pp. 821-835. doi: 10.1016/ j.actaastro.2009.08.033.

McGuire, M.K., Gage, P., Galloway, E.T., Huyhn, L., Nguyen, J., Bowles, J.V. and Windhorst, R., 2004, "Trajectory and thermal protection system design for reusable launch vehicles", AIAA Paper 2004-4490.
Menees, G.P., 1983, "Thermal protection requirements for near-Earth aeroassisted orbital transfer vehicle missions”, AIAA Paper 83-1513.

NASA Marshall Space Flight Center, 2003, "X-37 demonstrator to test future launch technologies in orbit and reentry environments", NASA Facts, FS-2003-05-65-MSFC.

Rajesh, K.A., 2002, "Reentry trajectory optimization: evolutionary approach”, AIAA Paper 2002-5466.

Reuther, J.J., Brown, J.L., Prabhu, D.K., McDaniel, R., Saunders, D. and Palmer, G., 2004, "External computational aerothermodynamic analysis of the Space Shuttle orbiter at STS-107 flight conditions", 37th AIAA Thermophysics Conference, Portland, Oregon, Paper no. AIAA- 2004-2281.

Tauber, M.E. and Sutton, K., 1991, "Stagnation-point radiative heating relations for Earth and Mars entries", Journal of Spacecraft, Vol. 28, No. 1, pp. 40-42. doi: 10.2514/3.26206.

Tran, H.K., Johnson, C.E., Rasky, D.J., Hui, F.C.L., Hsu, M.T., Chen, T., Chen, Y.K., Paragas, D. and Kobayashi, L., 1997, "Phenolic impregnated carbon ablators (PICA) as thermal protection systems for discovery missions", NASA TM-110440.

Vinh, N.X., Busemann, A. and Culp, R.D., 1980, "Hypersonic and planetary entry flight mechanics", University of Michigan Press, Ann Arbor, Ml.

Walberg, G.D., 1985, "A survey of aero-assisted orbit transfer", Journal of Spacecraft and Rockets, Vol. 22, No. 1, pp. 3-18. doi: 10.2514/3.25704.

Williams, S.D. and Curry, D.M., 1992, "Thermal protection materials thermophysical property data", NASA RP 1289.

Windhorst, R., Galloway, E., Lau, E., Saunders, D. and Gage, P., 2004, "Aerospace vehicle trajectory design and optimization within a multidisciplinary environment", AIAA Paper 2004-704.

Yeniay, Ö., 2005, "Penalty function methods for constrained optimization with genetic algorithms", Mathematical and Computational Applications, Vol. 10, No. 1, pp. 45-56. 


\section{LIST OF SYMBOLS}

$A=$ see Eq. (17)

$a, b=$ exponents, dependent on $\rho_{\text {stm }}$ and $V$

$B_{T}=$ activation temperature, $\mathrm{K}$

$C_{C}=$ constant, dependent on the atmosphere

$C_{0}=$ drag coefficient

$C_{D O}=$ zero-lift drag coefficient

$C_{L}=$ lift coefficient

$C_{\alpha} \alpha=$ derivative of $C$ wrt angle of attack

$C_{\text {max }}=$ maximum lift coefficient

$C_{r}=$ constant, dependent on the atmosphere

$c_{p}=$ specific heat, $\mathrm{J} /(\mathrm{kg} \cdot \mathrm{K})$

$D=$ drag, $\mathrm{N}$

$d t=$ integration time step, $\mathbf{s}$

$F=$ exterior view factor

$f(V)=$ function, dependent on the velocity

$f=$ fitness function

$g=$ gravitational acceleration, $\mathrm{m} / \mathrm{s}^{2}$

$g_{0}=$ gravitational acc. at sea level, $\mathrm{m} / \mathrm{s}^{2}$

$H=$ altitude, $m$

$H_{A}=$ altitude of the initial LEO, $m$

$H_{\text {etm }}=$ altitude of the sensible atmosphere, $\mathrm{m}$

$H_{B}=$ altitude of the final LEO, $m$

$H_{\min }=$ minimum flight altitude, $\mathrm{m}$

$H L=$ heat load, $\mathrm{J} / \mathrm{cm}^{2}$

$h=$ enthalpy, $\mathrm{J} / \mathrm{kg}$

$\bar{h}=$ partial heat of charring, $\mathrm{J} / \mathrm{kg}$

$h_{d}=$ pyrolysis enthalpy, $\mathrm{J} / \mathrm{kg}$

$h_{w}=$ wall enthalpy, $\mathrm{J} / \mathrm{kg}$

$h_{0}=$ total enthalpy, $\mathrm{J} / \mathrm{kg}$

$h_{s p}=$ propellant specific impulse, $\mathrm{s}$

$i=$ inclination, rad

$K_{c f}=$ collision frequency factor, $\mathrm{kg} /\left(\mathrm{m}^{3} \cdot \mathrm{s}\right)$

$K_{D}^{c f}=$ induced drag factor

$k=$ thermal conductivity, $\mathrm{W} /(\mathrm{m} \cdot \mathrm{K})$

$L=$ lift, $N$

$I=$ current length, see Figs. $6(a)$ and $6(b), m$

$I_{\text {ve }}=$ vehicle length, $\mathrm{m}$

$m=$ mass, $\mathrm{kg}$

$\dot{m}_{c}=$ char removal rate, $\mathrm{kg} /\left[\mathrm{m}^{2} \cdot \mathrm{s}\right]$

$\dot{m}_{g}^{c}=$ pyrolysis gas mass flow rate, $\mathrm{kg} /\left(\mathrm{m}^{2} \cdot \mathrm{s}\right]$

$\dot{m}_{\text {TPS }}=$ TPS mass, $\mathrm{kg}$

$m_{\text {TPS }}^{a b l}=$ mass of the ablative part of the TPS, $\mathrm{kg}$

$m_{T P S}^{s S}=$ mass of the TPS substructure, $\mathrm{kg}$

$m_{\text {TPS }}^{r e u}=$ mass of the reusable part of the TPS, $\mathrm{kg}$

$m_{\text {TPS los }}=$ TPS mass lost, $\mathrm{kg}$

$m_{\text {prop }}=$ propellant mass, $\mathrm{kg}$

$m_{v e, f i}=$ final vehicle mass, $\mathrm{kg}$

$m_{\text {ve, fin, ap }}=$ "all propulsive" case final mass, $\mathrm{kg}$

$m_{\text {ve, } i}=$ vehicle mass at atmospheric entry, $\mathrm{kg}$

$m_{v e, ~}=$ initial vehicle mass, $\mathrm{kg}$

$m_{v e, ~}=$ vehicle mass at atmospheric exit, $\mathrm{kg}$

$n=$ load factor, $g$

$n_{r}=$ decomposition reaction order

$\dot{Q}_{i n}=$ net total heat flux at surface, $\mathrm{W} / \mathrm{m}^{2}$

$\dot{Q}_{\text {in } \max }=$ maximum entering heat flux, $\mathrm{W} / \mathrm{m}^{2}$

$\dot{Q}_{\max }^{a b l}=$ max heat flux for ablative part, $\mathrm{W} / \mathrm{m}^{2}$

$\dot{Q}^{\text {reu }}=$ max allowable heat flux for reusable part, $\mathrm{W} / \mathrm{m}^{2}$

$q=$ dynamic pressure, $\mathrm{kN} / \mathrm{m}^{2}$

$\dot{q}_{c, b i w}=$ net hot wall convective heat flux, $\mathrm{W} / \mathrm{m}^{2}$

$\dot{q}_{\text {comb }}=$ combustion heat flux, $\mathrm{W} / \mathrm{m}^{2}$

$\dot{q}_{c o n}=$ net hot wall convective heat flux, $\mathrm{W} / \mathrm{m}^{2}$

$\dot{q}_{R}=$ internal radiative heat flux, $\mathrm{W} / \mathrm{m}^{2}$

$\dot{q}_{\text {rad }}=$ radiative heat flux, $\mathrm{W} / \mathrm{m}^{2}$

$\dot{q}_{t o t}=$ total heat flux, $\mathrm{W} / \mathrm{m}^{2}$

$R=$ radius, $\mathrm{m}$

$R_{A}=$ initial LEO radius, $\mathrm{m}$

$R_{\mathrm{atm}}=$ radius of the sensible atmosphere, $\mathrm{m}$

$R_{B}=$ final LEO radius, $m$

$R_{f, y}=$ reward factor for component ' $j$ '

$R_{\oplus}=$ Earth's radius, $\mathrm{m}$ $r_{n}=$ vehicle nose radius, $\mathrm{m}$

$r_{b}=$ vehicle body radius, $m$

$S=$ vehicle reference surface, $\mathrm{m}^{2}$

$\dot{s}_{r}=$ surface recession, $\mathrm{m}$

$s_{r}=$ char recession rate, $\mathrm{m} / \mathrm{s}$

$S_{T P S, v e}=$ vehicle TPS total surface, $\mathrm{m}^{2}$

$T$ = temperature, $\mathrm{K}$

$T_{B L}=$ bond-line temperature, $\mathrm{K}$

$T_{\text {L }}$ = bond-line limit temperature, $\mathrm{K}$

$T_{w}=$ wall temperature, $\mathrm{K}$

$T_{\infty}^{w}=$ freestream temperature, $\mathrm{K}$

$t=$ time, $\mathbf{s}$

$t_{f i}=$ flight time, $s$

$V=$ velocity modulus, $\mathrm{m} / \mathrm{s}$

$V_{A}=$ circular orbit speed in initial LEO, $\mathrm{m} / \mathrm{s}$

$V_{B}=$ circular orbit speed in final LEO, $\mathrm{m} / \mathrm{s}$

$V_{i}=$ speed at atmospheric entry, $\mathrm{m} / \mathrm{s}$

$V=$ speed at atmospheric exit, $\mathrm{m} / \mathrm{s}$

$w_{i j}=$ multiplicative weight of component ' $j$ '

$W C_{v e}=$ vehicle wing cord, $\mathrm{m}$

$w s_{v e}=$ vehicle wing span, $\mathrm{m}$

$x=$ mobile coordinate system, $y-S$, m; or current centerline abscissa, see Fig. 6 [a], m

$y=$ fixed coordinate system, $m$; or current wing ordinate, see Fig. 6 (b), m

$\Delta i=$ variation of the orbital inclination, rad

$\Delta h_{\text {comb }}=$ combustion heat per unit weight, $\mathrm{J} / \mathrm{kg}$

$\Delta V_{a p}=$ "all propulsive" impulse, $\mathrm{m} / \mathrm{s}$

$\Delta V_{1}=$ deorbit impulse, $\mathrm{m} / \mathrm{s}$

$\Delta V_{1, n}=$ minimum deorbit impulse, $\mathrm{m} / \mathrm{s}$

$\Delta V_{2}=$ boost impulse, $\mathrm{m} / \mathrm{s}$

$\Delta V_{3}=$ circularizing impulse, $\mathrm{m} / \mathrm{s}$

$\alpha=$ angle of attack, rad

$\alpha_{c}=$ weighting factor for char mass loss

$\alpha=$ weighting factor for pyrolysis gases

$\gamma=$ flight path angle, rad

$\gamma_{i}=$ flight path angle at atmospheric entry, rad

$\gamma_{u}=$ flight path angle at atmospheric exit, rad

$\delta_{\text {TPS }}=$ initial thickness of the ablative part, $\mathrm{mm}$

$\delta_{T P S}=$ initial thickness of the reusable part, $\mathrm{mm}$

$\varepsilon_{W}=$ surface emissivity

$\theta=$ longitude, rad

$\mu=$ gravitational parameter, $\mathrm{m}^{3} / \mathrm{s}^{2}$

$\rho=$ density, $\mathrm{kg} / \mathrm{m}^{3}$

$\rho_{\text {atm }}=$ atmospheric density, $\mathrm{kg} / \mathrm{m}^{3}$

$\sigma=$ bank angle, rad

$\sigma_{S B}=$ Stefan-Boltzmann constant, $W /\left[\mathrm{m}^{2} \cdot \mathrm{K}^{4}\right]$

$\varphi=$ latitude, $\mathrm{rad}$

$\psi=$ heading angle, rad

\section{Subscripts}

$A=$ value at initial LEO

$a p=$ "all propulsive"

$B=$ value at final LEO

$B L=$ bond-line

$C=$ char

$f l=$ flight

$g=$ pyrolysis gas

$H F=$ heat flux

$i=$ value at atmospheric entry

los $=$ lost

$m=$ mass

prop $=$ propellant

TPS = concerning Thermal Protection System

$u=$ value at atmospheric exit

$v=$ virgin material

$v e=$ vehicle

$w=$ wall

$\Delta i=$ inclination variation

Superscripts

$a b l=$ ablative

$s s=$ substructure

reu $=$ reusable 